\title{
Image Acquisition
}

National Cancer Institute

\section{Source}

National Cancer Institute. Image Acquisition. NCI Thesaurus. Code C43385.

The process by which an image is acquired or obtained. 